\title{
Minimum Total Noise in Wave-Mixing Processes
}

\author{
Savita Gill, ${ }^{1}$ Sunil Rani, ${ }^{2}$ and Nafa Singh $^{3}$ \\ ${ }^{1}$ Department of Applied Physics, University Institute of Engineering and Technology, Kurukshetra 136 119, India \\ ${ }^{2}$ Department of Applied Physics, S.K. Institute of Engineering and Technology, Kurukshetra 136 118, India \\ ${ }^{3}$ Department of Physics, Kurukshetra University, Kurukshetra 136 119, India
}

Correspondence should be addressed to Savita Gill, savitamaggie@gmail.com

Received 1 November 2011; Accepted 9 February 2012

Academic Editor: Nicusor Iftimia

Copyright (C) 2012 Savita Gill et al. This is an open access article distributed under the Creative Commons Attribution License, which permits unrestricted use, distribution, and reproduction in any medium, provided the original work is properly cited.

Higher-order squeezing in different optical processes such as seven-wave mixing and five-wave mixing has been studied. The total noise of a field state is a measure of the fluctuations of the field amplitude. It is shown that the minimum total noise ( $\left.T_{\min }\right)$ of a higher-order squeezed state always increases with the increase in nonclassicality associated with higher-order squeezing. Thus, from $T_{\min }$, one can conclude that highly nonclassical states have large amplitude fluctuations.

\section{Introduction}

The concept of the total noise of a quantum state was introduced by Schumaker [1]. As was pointed by Schumaker, the total noise is always greater than or equal to a half and reaches this value only for coherent states. The total noise of a field state increases as the depth of nonclassicality associated with a state increases [2]. A nonclassical state of electromagnetic field is one for which the Glauber-Sudarshan P-function either goes negative or contains derivatives of delta function [3]. Standard deviation of an observable is considered to be the most natural measure of quantum fluctuations associated with an observable [4]. Reduction of quantum fluctuation below the coherent state level corresponds to a nonclassical state. Optical fields in states with purely quantum mechanical properties are the key ingredients of quantum optics. Nonclassical properties of a radiation field such as photon antibunching and squeezing are currently of great interest and have attracted considerable attention owing to its low noise property [5-9]. Higher-order squeezing has drawn the greater attention of the community due to the rapid development of techniques for making higher-order correlation measurements in quantum optics [10-15].

In the present work, we have reported that the generation of higher-order squeezed state is possible by using sevenwave mixing and five-wave mixing processes, respectively.
Further, we have also shown that $T_{\min }$ can be used as an indirect measure of nonclassicality of a system associated with higher-order squeezing.

\section{Higher-Order Squeezing and Total Noise}

Higher-order squeezing is defined in various ways. Hong and Mandel [10] and Hillery [12] have introduced the notion of higher-order squeezing of quantized electromagnetic field as generalization of normal squeezing. Amplitude-squared squeezing is defined in terms of operators $Y_{1}$ and $Y_{2}$ as

$$
Y_{1}=\frac{1}{\sqrt{2}}\left(A^{2}+A^{\dagger 2}\right), \quad Y_{2}=\frac{i}{\sqrt{2}}\left(A^{2}-A^{\dagger 2}\right),
$$

where $Y_{1}$ and $Y_{2}$ are the real and imaginary parts of the square of field amplitude, respectively. $A$ and $A^{\dagger}$ are slowly varying operators defined by $A=a e^{i \omega t}$ and $A^{\dagger}=a^{\dagger} e^{-i \omega t}$.

The operators $Y_{1}$ and $Y_{2}$ obey the commutation relation

$$
\left[Y_{1}, Y_{2}\right]=i(4 N+2) \text {. }
$$

This leads to the uncertainty relation

$$
\Delta Y_{1} \Delta Y_{2} \geq\langle(2 N+1)\rangle
$$

where $N$ is the usual number operator.

Amplitude-squared squeezing is said to exist in $Y_{1}$ variable if

$$
\left(\Delta Y_{1}\right)^{2}<\langle 2 N+1\rangle
$$


or the squeezing $f$ is

$$
f=\left(\Delta Y_{1}\right)^{2}-\langle 2 N+1\rangle<0 .
$$

Total noise of a quantum state of a single mode, having density matrix $\rho$, is defined as

$$
T(\rho)=\left(\Delta X_{1}\right)^{2}+\left(\Delta X_{2}\right)^{2} .
$$

Increased nonclassicality gives rise to increase in the total noise. This fact can be verified by associating total noise with higher-order squeezing. The uncertainty relations for the quadrature variables using Hillery's approach [2] may be written as follows:

$$
\begin{aligned}
& \left(\Delta X_{1}\right)^{2}\left(\Delta Y_{1}\right)^{2} \geq \frac{1}{2}\left\langle X_{2}\right\rangle^{2}, \\
& \left(\Delta X_{2}\right)^{2}\left(\Delta Y_{1}\right)^{2} \geq \frac{1}{2}\left\langle X_{1}\right\rangle^{2} .
\end{aligned}
$$

We can combine the above relations with the identity

$$
\langle 2 N+1\rangle=\left\langle X_{1}\right\rangle^{2}+\left\langle X_{2}\right\rangle^{2}
$$

to obtain

$$
\begin{aligned}
\frac{1}{2}(\langle 2 N+1\rangle) & =\frac{1}{2}\left(\left\langle X_{1}\right\rangle^{2}+\left\langle X_{2}\right\rangle^{2}\right) \\
& \leq\left(\left(\Delta X_{1}\right)^{2}+\left(\Delta X_{2}\right)^{2}\right)\left(\Delta Y_{1}\right)^{2}
\end{aligned}
$$

or

$$
T=\left(\Delta X_{1}\right)^{2}+\left(\Delta X_{2}\right)^{2} \geq \frac{1}{2} \frac{(\langle 2 N+1\rangle)}{\left(\Delta Y_{1}\right)^{2}} .
$$

From condition (4), $T$ will be greater than $1 / 2$. The minimum total noise of a state is greater as a state becomes more nonclassical:

$$
T_{\min }=\frac{1}{2} \frac{(\langle 2 N+1\rangle)}{\left(\Delta Y_{1}\right)^{2}}
$$

Here again we see that, for fixed $\langle N\rangle$ as $\Delta Y_{1}$ decreases, the total noise must increase. Thus, $T_{\min }$ increases as a state becomes more squeezed and may be considered as a measure of depth of nonclassicality.

\section{Seven-Wave Mixing Process}

In this process, the interaction is looked upon as a process which involves the absorption of two pump photons, each having frequency $\omega_{1}$ and emission of two probe photons of frequency $\omega_{2}$, and three signal photons of frequency $\omega_{3}$ where

$$
2 \omega_{1}=2 \omega_{2}+3 \omega_{3} .
$$

The Hamiltonian for this process is given as follows $(\hbar=1)$ :

$$
H=\omega_{1} a^{\dagger} a+\omega_{2} b^{\dagger} b+\omega_{3} c^{\dagger} c+g\left(a^{2} b^{\dagger 2} c^{\dagger 3}+a^{\dagger 2} b^{2} c^{3}\right),
$$

in which $g$ is a coupling constant. $A=a \exp \left(i \omega_{1} t\right), B=b \exp$ $\left(i \omega_{2} t\right)$, and $C=c \exp \left(i \omega_{3} t\right)$ are the slowly varying operators at frequencies $\omega_{1}, \omega_{2}$, and $\omega_{3}, a\left(a^{\dagger}\right), b\left(b^{\dagger}\right)$, and $c\left(c^{\dagger}\right)$ are the usual annihilation (creation) operators, respectively. The Heisenberg equation of motion for fundamental mode $A$ is given as $(\hbar=1)$ :

$$
\frac{d A}{d t}=\frac{\partial A}{\partial t}+i[H, A]
$$

By using the short-time approximation technique, we expand $A(t)$ by using Taylors series expansion and retaining the terms up to $g^{2} t^{2}$ as

$$
\begin{gathered}
A(t)=A-2 i g t A^{\dagger} B^{2} C^{3}+g^{2} t^{2} \\
\times\left[2 A N_{B}^{2} N_{C}^{3}-9 A^{\dagger} A^{2} N_{B}^{2} N_{C}^{2}-18 A^{\dagger} A^{2} N_{B}^{2} N_{C}\right. \\
-6 A^{\dagger} A^{2} N_{B}^{2}-4 A^{\dagger} A^{2} N_{B} N_{C}^{3}-36 A^{\dagger} A^{2} N_{B} N_{C}^{2} \\
-72 A^{\dagger} A^{2} N_{B} N_{C}-24 A^{\dagger} A^{2} N_{B}-2 A^{\dagger} A^{2} N_{C}^{3} \\
\left.-18 A^{\dagger} A^{2} N_{C}^{2}-36 A^{\dagger} A^{2} N_{C}-12 A^{\dagger} A^{2}\right],
\end{gathered}
$$

where $N_{A}=A^{\dagger} A, N_{B}=B^{\dagger} B$, and $N_{C}=C^{\dagger} C$.

Initially, we consider the quantum state of the field amplitude as a product of coherent state for the fundamental mode $A$ and the vacuum state for modes $B$ and $C$, that is,

$$
|\psi\rangle=|\alpha\rangle_{A}|0\rangle_{B}|0\rangle_{C} .
$$

Using (1), (15), and (16), a straightforward but strenuous calculation yields

$$
\begin{aligned}
{\left[\Delta Y_{1 A}(t)\right]^{2}=} & \left\langle\psi\left|Y_{1 A}^{2}(t)\right| \psi\right\rangle-\left\langle\psi\left|Y_{1 A}(t)\right| \psi\right\rangle^{2} \\
= & 2|\alpha|^{2}+1 \\
& -12 g^{2} t^{2}\left[2|\alpha|^{4}(\cos 4 \theta+3)+10|\alpha|^{2}+2\right] .
\end{aligned}
$$

Using (15) and (16), number of photons in mode $A$ may be expressed as

$$
\begin{aligned}
N(t) & =A^{\dagger}(t) A(t) \\
& =|\alpha|^{2}-24 g^{2} t^{2}|\alpha|^{4} .
\end{aligned}
$$

Now, we can substitute (17) and (18) in (5) to obtain a closed form analytic expression for $f$ as

$$
\begin{aligned}
f & =\left(\Delta Y_{1}\right)^{2}-\langle 2 N+1\rangle \\
& =-12 g^{2} t^{2}\left[2|\alpha|^{4}(\cos 4 \theta+1)+10|\alpha|^{2}+2\right] .
\end{aligned}
$$

And similarly substituting (17) and (18) in (11), we have

$$
\begin{aligned}
T_{\min } & =\frac{1}{2} \frac{(\langle 2 N+1\rangle)}{\left(\Delta Y_{1}\right)^{2}} \\
& =\frac{1}{2} \frac{2|\alpha|^{2}+1-48 g^{2} t^{2}|\alpha|^{4}}{2|\alpha|^{2}+1-12 g^{2} t^{2}\left[2|\alpha|^{4}(\cos 4 \theta+3)+10|\alpha|^{2}+2\right]},
\end{aligned}
$$

where $\theta$ is the phase angle, with $\alpha=|\alpha| e^{i \theta}$. The right-hand side of (19) is negative and thus indicating the presence of higher-order squeezing within the short time domain of the second-order solution. 


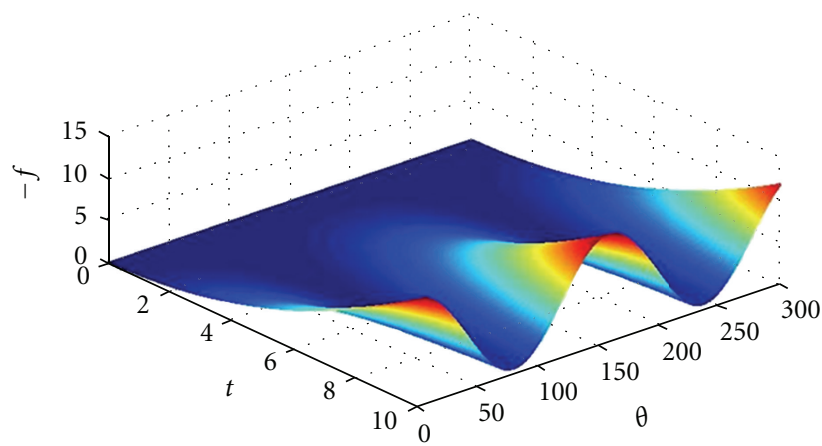

(a)

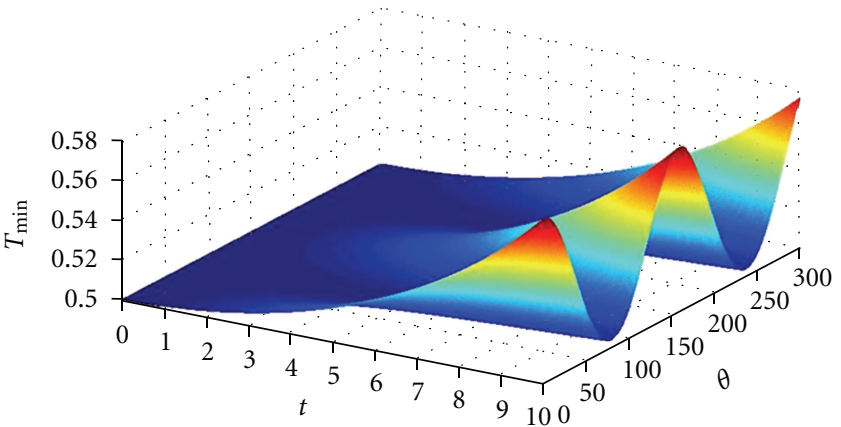

(b)

FIGURE 1: (a) Variation of $-f$ for seven-wave mixing process with respect to interaction time $t$ and initial phase of the coherent state $\theta$. (b) Variation of $T_{\min }$ of seven-wave mixing process with respect to interaction time $t$ and initial phase of the coherent state $\theta$.

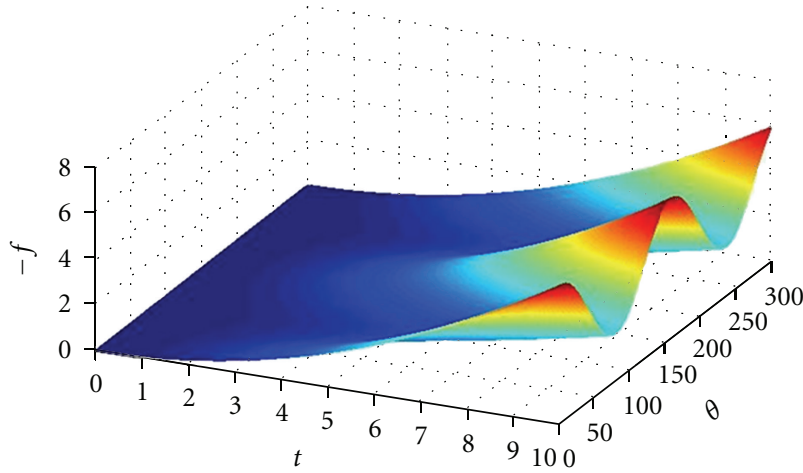

(a)

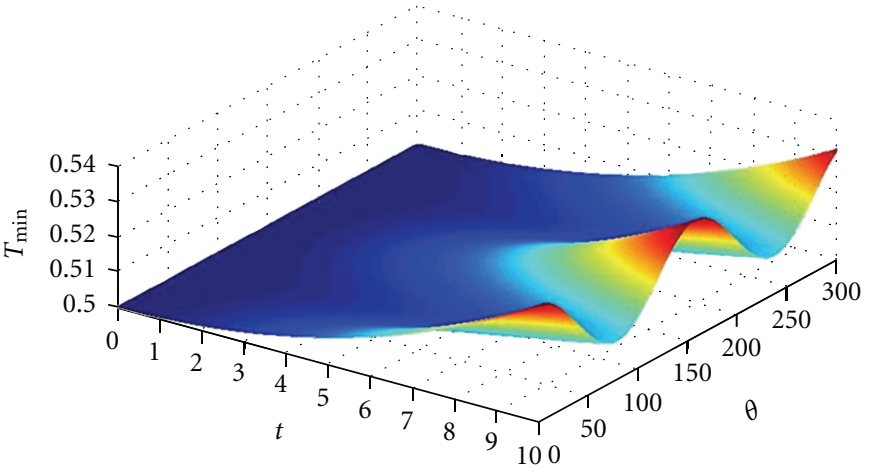

(b)

Figure 2: (a) Variation of $-f$ for five-wave mixing process with respect to interaction time $t$ and initial phase of the coherent state $\theta$. (b) Variation of $T_{\min }$ of five-wave mixing process with respect to interaction time $t$ and initial phase of the coherent state $\theta$.

\section{Five-Wave Mixing Process}

In this process, the interaction is looked upon as a process which involves the absorption of two pump photons, each having frequency $\omega_{1}$ and emission of two probe photons of frequency $\omega_{2}$ and signal photon of frequency $\omega_{3}$, where

$$
2 \omega_{1}=2 \omega_{2}+\omega_{3}
$$

The Hamiltonian for this process is given as follows $(\hbar=1)$ :

$$
H=\omega_{1} a^{\dagger} a+\omega_{2} b^{\dagger} b+\omega_{3} c^{\dagger} c+g\left(a^{2} b^{\dagger 2} c^{\dagger}+a^{\dagger 2} b^{2} c\right) .
$$

Short-time second-order solution of this Hamiltonian is

$$
\begin{aligned}
A(t)= & A-2 i g t A^{\dagger} B^{2} C+g^{2} t^{2} \\
\times & {\left[2 A N_{B}^{2} N_{C}-A^{\dagger} A^{2} N_{B}^{2}-4 A^{\dagger} A^{2} N_{B} N_{C}\right.} \\
& \left.-4 A^{\dagger} A^{2} N_{B}-2 A^{\dagger} A^{2} N_{C}-2 A^{\dagger} A^{2}\right],
\end{aligned}
$$

where $N_{A}=A^{\dagger} A, N_{B}=B^{\dagger} B$, and $N_{C}=C^{\dagger} C$.
Using (23) and (16), a straightforward but strenuous calculation yields

$$
\begin{aligned}
{\left[\Delta Y_{1 A}(t)\right]^{2}=} & \left\langle\psi\left|Y_{1 A}^{2}(t)\right| \psi\right\rangle-\left\langle\psi\left|Y_{1 A}(t)\right| \psi\right\rangle^{2} \\
= & 2|\alpha|^{2}+1 \\
& -4 g^{2} t^{2}\left[2|\alpha|^{4}(\cos 4 \theta+3)+8|\alpha|^{2}+1\right] .
\end{aligned}
$$

Using (23) and (16), number of photons in mode $A$ may be expressed as

$$
\begin{aligned}
N_{1 A}(t) & =A^{\dagger}(t) A(t) \\
& =|\alpha|^{2}-4 g^{2} t^{2}|\alpha|^{4} .
\end{aligned}
$$

The respective values of $f$ and $T_{\text {min }}$ can similarly be calculated as we have done for seven-wave mixing and that yields

$$
\begin{aligned}
f & =\left(\Delta Y_{1}\right)^{2}-\langle 2 N+1\rangle \\
& =-4 g^{2} t^{2}\left[2|\alpha|^{4}(\cos 4 \theta+2)+8|\alpha|^{2}+1\right] \\
T_{\min } & =\frac{1}{2} \frac{2|\alpha|^{2}+1-48 g^{2} t^{2}|\alpha|^{4}}{2|\alpha|^{2}+1-12 g^{2} t^{2}\left[2|\alpha|^{4}(\cos 4 \theta+3)+10|\alpha|^{2}+2\right]} .
\end{aligned}
$$


The right-hand side of (26) is always negative within the domain of the validity of the solution which shows the existence of higher-order squeezing.

\section{Results}

The presence of higher-order squeezing in seven-wave mixing and five-wave mixing processes is being shown in (19) and (26), respectively. Again, from (5), (10), and (11), it is clear that highly nonclassical states have large amplitude fluctuations. Taking $g^{2}=10^{-6}$ and $|\alpha|^{2}=50$, the variation of squeezing $(-f)$ and minimum total noise $\left(T_{\min }\right)$ in sevenwave mixing and five-wave mixing processes are shown in Figures 1 and 2, respectively.

The degree of higher-order squeezing varies with the phase of the input coherent light $\theta$, initial photon number $|\alpha|^{2}$, and the interaction time $t$. Further, Figures $1(\mathrm{~b})$ and 2(b) show that the depth of nonclassicality in higher-order squeezing can be measured in terms of $T_{\min }$.

\section{Conclusion}

The results show the presence of higher-order squeezing in seven-wave mixing and five-wave mixing processes. The total noise which is a measure of the size of amplitude fluctuations of a state of the field always increases with the increase in nonclassicality of a system associated with higher-order squeezing. This fact is more conspicuous in Figures 1 and 2. Again, from (19), (20), (26), and (27), we can conclude that squeezing and the amount of total noise present in the system can be tuned by varying the values of initial phase of the coherent state $(\theta)$, number of photons present in the radiation field prior to the interaction $\left(|\alpha|^{2}\right)$, and the interaction time $(t)$.

\section{References}

[1] B. L. Schumaker, "Quantum mechanical pure states with gaussian wave functions," Physics Reports, vol. 135, no. 6, pp. 317-408, 1986.

[2] M. Hillery, "Total noise and nonclassical states," Physical Review A, vol. 39, no. 6, pp. 2994-3002, 1989.

[3] P. Meystre and M. Sargent III, Elementof Quantum Optics, Springer, Berlin, Germany, 1991.

[4] A. Orlowski, "Classical entropy of quantum states of light," Physical Review A, vol. 48, no. 1, pp. 727-731, 1993.

[5] T. Eberle, S. Steinlechner, J. Bauchrowitz et al., "Quantum enhancement of the zero-area sagnac interferometer topology for gravitational wave detection," Physical Review Letters, vol. 104, no. 25, Article ID 251102, 2010.

[6] H. Vahlbruch, A. Khalaidovski, N. Lastzka, C. Gräf, K. Danzmann, and R. Schnabel, "The GEO 600 squeezed light source," Classical and Quantum Gravity, vol. 27, no. 8, Article ID 084027, 2010.

[7] N. Takei, T. Aoki, S. Koike et al., "Experimental demonstration of quantum teleportation of a squeezed state," Physical Review A, vol. 72, no. 4, Article ID 042304, 2005.

[8] J. Calsamiglia, M. Aspachs, R. Muñoz-Tapia, and E. Bagan, "Phase-covariant quantum benchmarks," Physical Review A, vol. 79, no. 5, Article ID 050301, 2009.
[9] J. S. Neergaard-Nielsen, B. M. Nielsen, C. Hettich, K. Mølmer, and E. S. Polzik, "Generation of a superposition of odd photon number states for quantum information networks," Physical Review Letters, vol. 97, no. 8, Article ID 083604, 2006.

[10] C. K. Hong and L. Mandel, "Higher-order squeezing of a quantum field," Physical Review Letters, vol. 54, no. 4, pp. 323325, 1985.

[11] C. K. Hong and L. Mandel, "Generation of higher-order squeezing of quantum electromagnetic fields," Physical Review A, vol. 32, no. 2, pp. 974-982, 1985.

[12] M. Hillery, "Amplitude-squared squeezing of the electromagnetic field," Physical Review A, vol. 36, no. 8, pp. 3796-3802, 1987.

[13] S. Rani, J. Lal, and N. Singh, "Squeezing up to fourth-order in the pump mode of eight-wave mixing process," Optical and Quantum Electronics, vol. 39, no. 9, pp. 735-745, 2007.

[14] S. Rani, J. Lal, and N. Singh, "Squeezing and sub-Poissonian effects up-to fourth order in fifth harmonic generation," Optics Communications, vol. 281, no. 2, pp. 341-346, 2008.

[15] D. K. Giri and P. S. Gupta, "Higher-order squeezing of the electromagnetic field in spontaneous and stimulated Raman processes," Journal of Modern Optics, vol. 52, no. 12, pp. 17691781, 2005. 

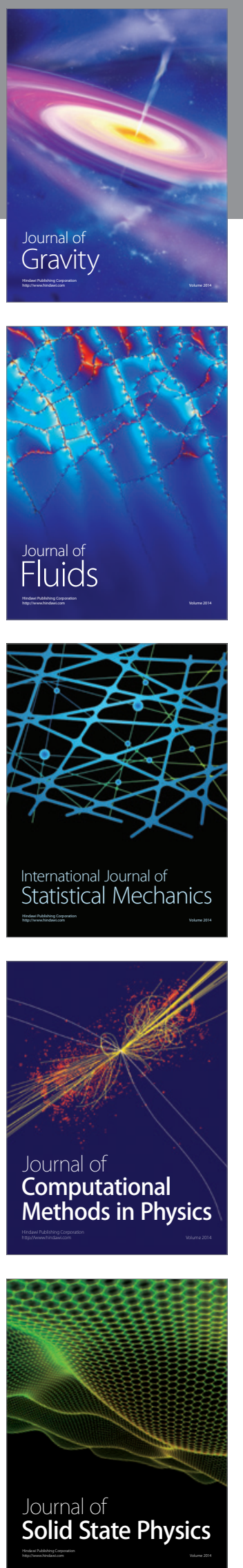

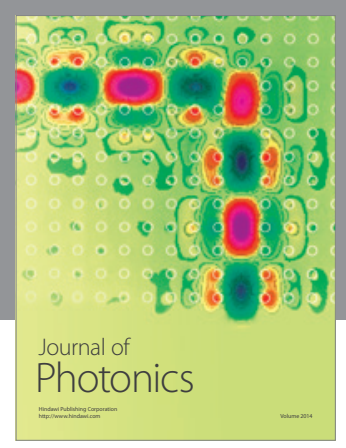

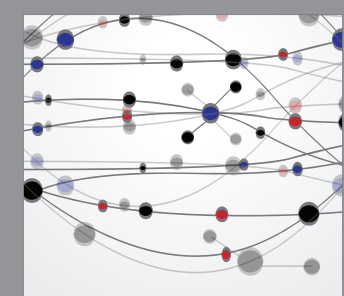

The Scientific World Journal
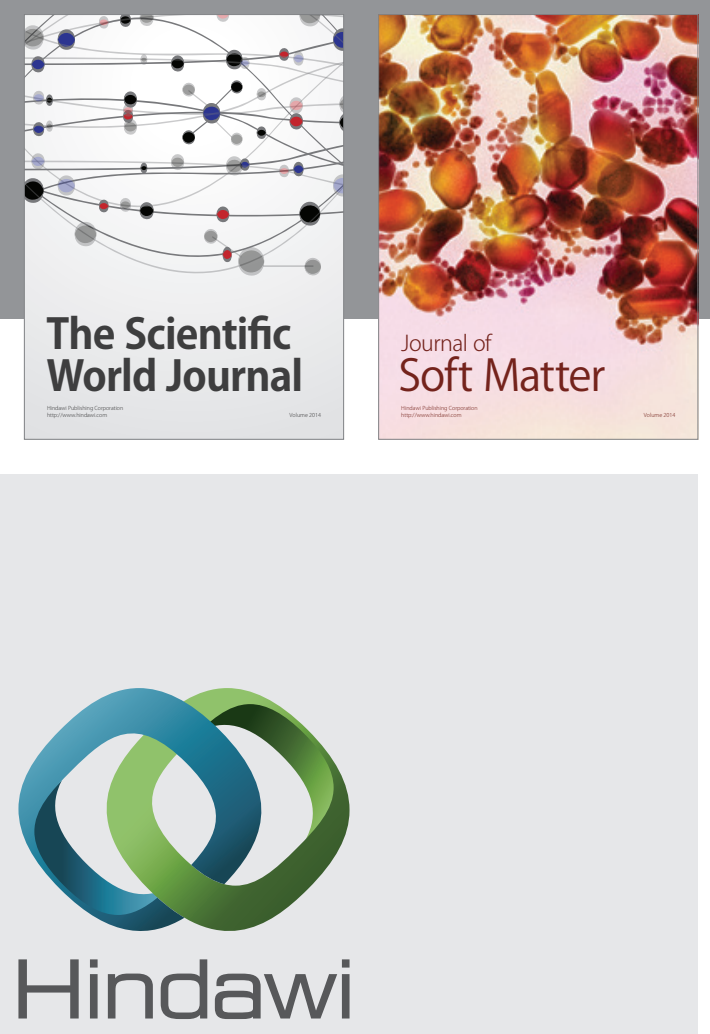

Submit your manuscripts at

http://www.hindawi.com
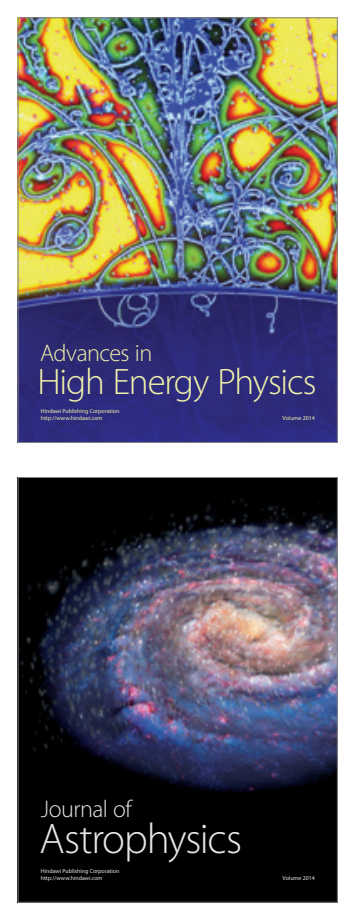
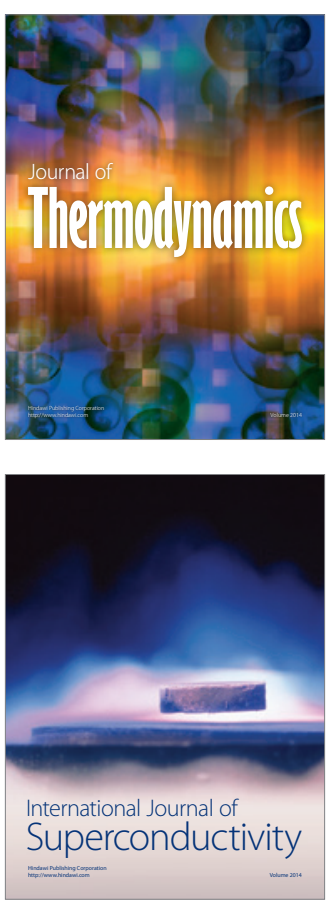
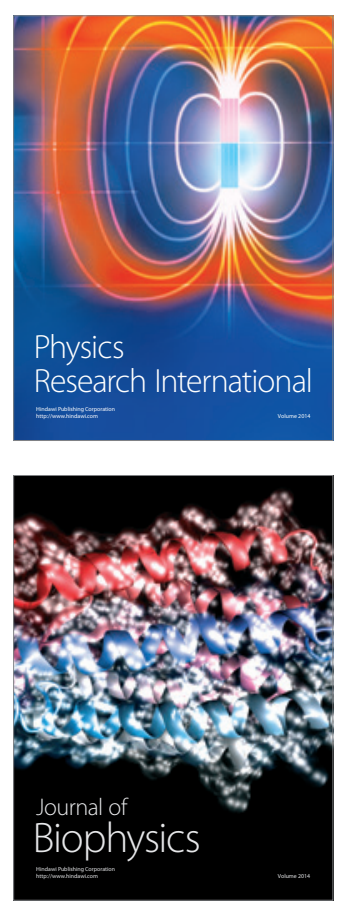
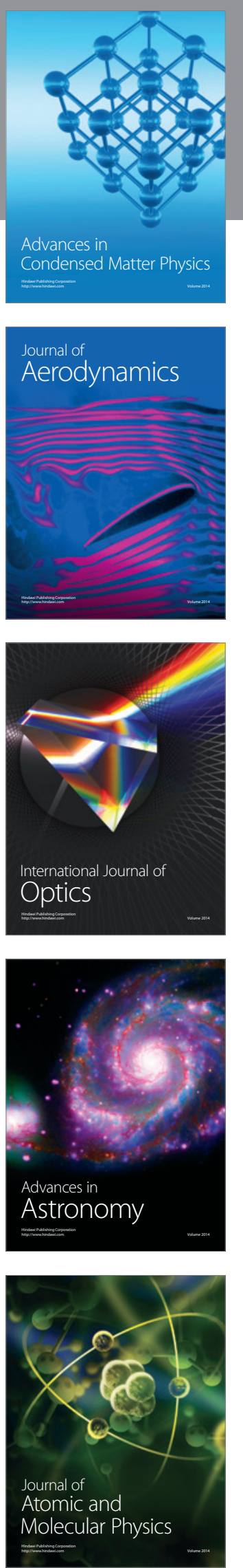